Onkologie 1984;7:389-391

\title{
Inhalt, Vol. 7, 1984
}

Kayser, K.; Burkhardt, H. U. und Doerr, W. , Heidelberg, Bleyl, U., Mannheim, Diezel, P. B., Pforzheim,

Gusek, W.; Schneider, W. und Wolfmüller, W., Karlsruhe, und Holch, P., Heilbronn

Bevölkerungsbezogene Daten in Nordbaden über bös-

artige Geschwülste des Magen-Darm-Traktes

\section{4}

Aigner, K. R.; Walther, H.; Tonn, J. C; Link, K. H.;

Schoch, P. und Schwemmle, K., Gießen

Die isolierte Leberperfusion bei fortgeschrittenen Meta

stasen kolorektaler Karzinome $\quad 13$

Wagner, T., Lübeck

Die Pharmakokinetik von 5-Fluorouracil und seine Per meation in Pleuraergüsse bei der Therapie des metastasierenden Mamma-Karzinoms 22

Sonderbände 26

Herzog, R. E. und Pellicer, A., Mainz Histographische DNS-Muster als Hilfe bei Diagnostik und Therapie des frühinvasiven Endometriumkarzinoms . 27

Schaar, H.; Schedel, I.; Schiemann, U. und Deicher, H., Hannover

Klinische Stadieneinteilung und Verlaufsbeurteilung von Patienten mit multiplem Myelom und benigner monoklonaler Hyperglobulinämie und Hilfe der Gesamttumor-

zellmassenberechnung nach Salmon und Wampler .... 32

Neuigkeiten für die klinische Praxis 40

Görg, C; Görg, K.; Gropp, C. und Havemann, K., Marburg

Lokalisiertes Ödem nach zytostatischer Therapie $\quad 42$

Matthias, M.; Sohr, R.; Preiß, R. und Brockmann, B.,

Berlin

Untersuchungen zur Bioverfügbarkeit von Cyclophos-

phamid nach peroraler Applikation im hohen Dosis-

bereich 48

Buchbesprechungen 50

Onkologie-Mitteilungen

Vorwort 62

Kleeberg, U.R., Hamburg

Geriatrische Aspekte der Onkologie 63

Fliedner, T. M. , Ulm

Gedanken zur Physiologie und Pathophysiologie des 


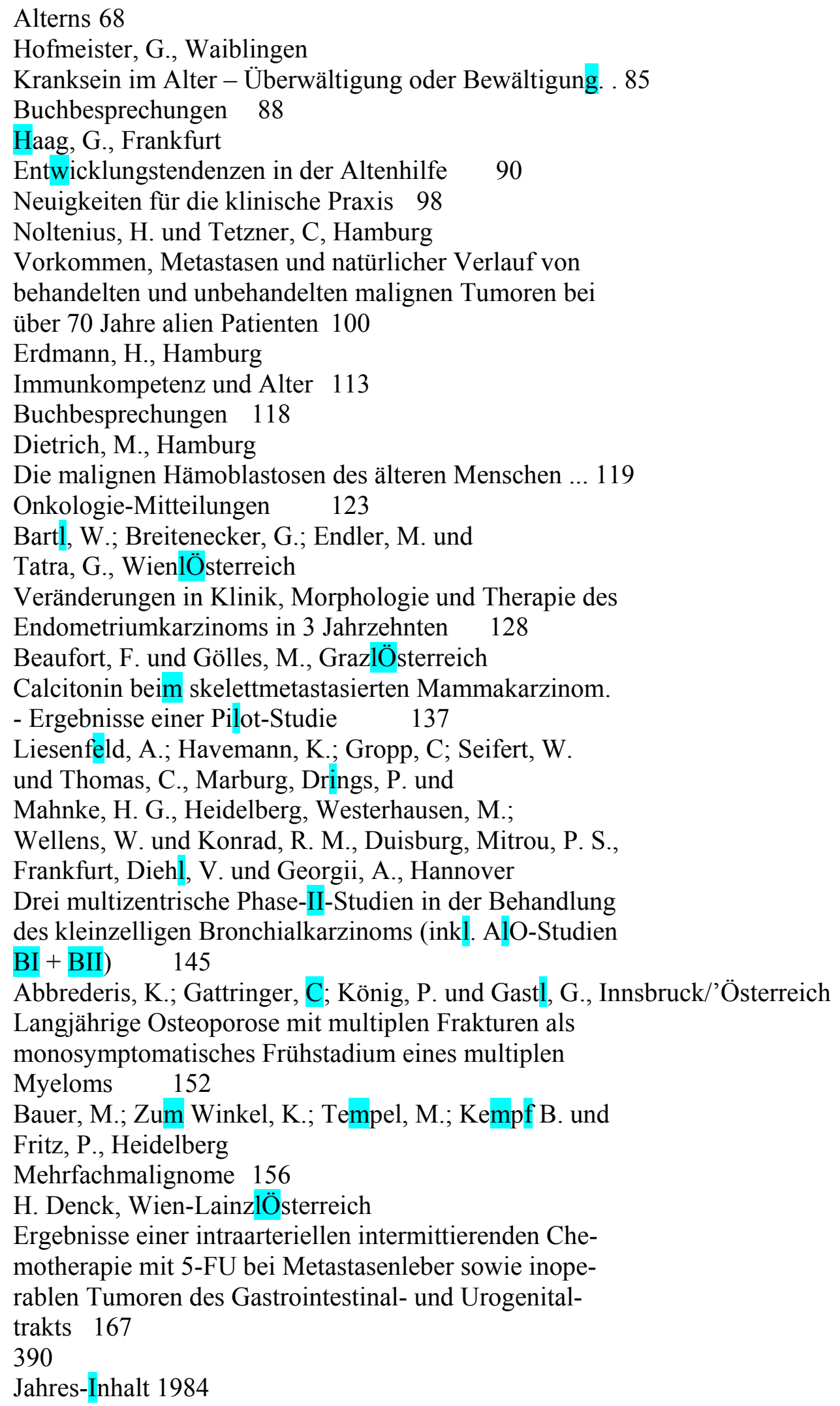


Neuigkeiten für die klinische Praxis 179

Buchbesprechungen 182

Von Heyden, H.-W.; Schroder, M.; Scherpe, A.;

Beyer, J.-H.; Kaboth, U.; Stennert, E. und Nagel, G. A.;

Göttingen, Gerhartz, H.; Foth, B. und Kastenbauer, E.,

Berlin, Westerhausen, M. und Schroeder, M., Duisburg,

Caliebe, W.; Rudert, H. und Liffers, R., Kiel,

Hofmann, J. und Schneider, B., Hannover

Zytostatische Therapie von fortgeschrittenen Plattenepithelkarzinomen des Kopf-Hals-Bereiches. - Eine randomisierte Studie zum Vergleich von cis-Dichlorodiaminoplatinum-(П) (cis-DDP) und Bleomycin (BLM) gegen

Methotrexat (MTX) und Vindesin (VDS) 183

Buchbesprechung 192

Onkologie-Mitteilungen 194

Drings, P.; Kleckow, M.; Manke, H. G. und Stiefel, E.,

Heidelberg

Chemotherapie des fortgeschrittenen nicht-kleinzelligen

Bronchialkarzinoms mit Cisplatin und Vindesin 202

Müller-Weihrich, St., München; Henze, G.;

Langermann, H. J.; Odenwald, E, und Riehm, H., Berlin

Kindliche B-Zell-Lymphome und -Leukämien. - Verbesserung der Prognose durch eine für B-Neoplasien konzipierte Therapie der BFM-Studiengruppe 205

Heidemann, E. und Dietz, K., Tubingen;

Obert, H. J., Laupheim, und Wilms, K., Würzburg

Günstigerer Verlauf des Herpes zoster bei immunsupprimierten Patienten unter Behandlung mit Fibroblasten-

interferon 210

Hiddemann, W.; Urbanitz, D.; Preusser, P.;

Balleisen, L.; Kirchhof, B.; Stenzinger, W. und

Büchner, Th., Münster; Achterrath, W., Neu-Issenburg

Kombinationstherapie mit AMSA und Etoposid (VP 16-

213) bei refraktärer akuter myeloischer Leukämie:

Eine Phase-I-Studie 214

Strauch, St. und Schellong, G., Münster;

Wannenmacher, M., Freiburg

Abgestufte Chemotherapie und reduzierte Strahlenthera-

pie beim Morbus Hodgkin im Kindesalter. - Zwischenbe-

richt der kooperativen Therapiestudie HD 82217

Buchbesprechung 221

Breitenecker, G.; Bartl, W.; Endler, M. und Gring, H., Wien

Die prognostische Bedeutung morphologischer Para

meter bei Endometriumkarzinomen 222

Sonderbände 235 
Neuigkeiten für die klinische Praxis 236

Städtler, R.; von Kries, R. und Göbel, U., Düsseldorf

Plättchenfunktion und hochdosierte Methotrexat-

Behandlung mit Citrovorumfaktor-Rescue 238

Kucera, H.; Wagner, G. und Weghaupt, K. , Wien

Komplikationen der präoperativen High-dose-rate-

Iridium-192-Vorbestrahlung beim Gebärmutterhals-

karzinom 244

Onkologie-Mitteilungen 253

K. O. Schmid, GrazlÖsterreich

Histologische Subtypen des Bronchuskarzinoms und

deren biologisches Verhalten 260

Sonderbände 262

Denck, H. und Kutschera, W., Wien-Lainz/Österreich

Das Bronchialkarzinom aus chirurgischer Sicht

A. Hackl, GrazlÖsterreich

Die Therapie des Bronchuskarzinoms aus der Sicht des

Strahlentherapeuten 268

Rab, B. und Sabitzer, H., KlagenfurtlÖsterreich

Co-Referat $\mathrm{zu} »$ Therapie des Bronchialkarzinoms aus der

Sicht des Strahlentherapeuten« 273

H. Rainer, WienlÖsterreich

Chemotherapie des kleinzelligen Bronchuskarzinoms . 276

O. Kokron, Wien-Lainz/Österreich

Chemotherapie des nicht-kleinzelligen Bronchuskarzi

noms 285

Jakse, R. und Lehnerl, M., GrazlÖsterreich

Biologisches Tumorverhalten und kurative Primär-

therapie bei HNO-Tumoren 293

Lehnert, M.; Jakse, R. und Seewann, H. L.,

GrazlÖsterreich

Chemotherapie bei fortgeschrittenem Plattenepithel-

karzinom des HNO-Bereiches 297

Scherpe, A.; Schroder, M.; von Heyden, H.-W. und Nagel, G. A., Göttingen

Chemotherapieergebnisse mit Cisplatin und 5-Fluoro-

uracil bei Plattenepithelkarzinomen des Kopf-Hals-

Bereiches 302

Gatzemeier, U.; Zschaber, R.; Hossfeld, D. K. und

Radenbach, D., Hamburg

Cisplatin, Vindesin und VP 16-213 in der Behandlung des

Jahres-Inhalt 1984

391

inoperablen nicht-kleinzelligen Bronchialkarzinoms. -

Eine klinische Phase-II-Studie $\quad 308$

Neuigkeiten für die klinische Praxis 316 
Buchbesprechungen 318

Onkologie-Mitteilungen $\quad 320$

Kleeberg, U. R., Hamburg-Altona

Editorial $\quad 326$

Treidler, J.; Pompecki, R.; Müllerleíle, U.; Garbrecht, M. und Kleeberg, U. R. , Hamburg

Prognostische Aussage des Serum-CEA und therapie-

bedingte unspezifische CEA-Verläufe beim metastasie-

renden Mammakarzinom 328

Buchbesprechungen 334

Friedmann, W.; Steffens, J. und Lobeck, H., Berlin

Immunhistochemische Diagnose des metastasierenden

Prostatakarzinoms 337

Jehn, U. und Mezger, J., München

Phase-II-Studie zur Behandlung der CML-Blastenkrise

mit Vindesin und Prednison 342

Buchbesprechungen 346

Aigner, K, R.; Jungbluth, A.; Link, K. H.; Walther, H.;

Müller, H.; Schwemmle, K.; Ringenberg, Th.;

Börger, G.; Ruppel, R. und Illig, L., Gießen; Voigt, H.,

Hamburg-Altona

Die isolierte hypertherme Extremitätenperfusion mit

Vindesin, Dacarbazin und Cis-Platin bei der Behandlung

maligner Melanome 348

Buchbesprechungen 354

Neuigkeiten für die klinische Praxis 356

Görg, C; Görg, K.; Gropp, C. und Havemann, K.,

Marburg

Aggressive Chemotherapie bei generalisiertem eosino-

philen Granulom im Erwachsenenalter $\quad 360$

Wander, H.-E., Göttingen

Panmyelopathie unter Aminoglutethimid. - Ein Fall-

bericht 367

Fiebig, H. H.; Henß, H.; Arnold, H. und Löhr, G. W., Freiburg; Wellens, W.,

DuisburglRegensburg; Westerhausen, M., Duisburg und Peukert, M., Bielefeld Phase-II-Studie des wasserlöslichen Nitrosoharnstoffes ACNUbeifortgeschrittenen kolorektalenKarzinomen. .

370

Petersen, K. G.; Arnold, H.i Lehmann, M. undKerp, L.,

Freiburg

Reversible orthostatische Hypotension unter Vincristin-

therapie $\quad 378$

Baczako, K. und Fischer, H., Ulm

Das maligne metastasierende Cystosarkoma phylloides

der Mamma. - Ulmer Sammlung seltener Tumoren: VI. . 382

Onkologie-Mitteilungen $\quad 385$

Jahres-Inhalt $1984 \quad 389$

Autorenverzeichnis $1984 \quad 392$ 